\title{
COVID-19 confirmed patients with negative antibodies results
}

\author{
Jian Wang ${ }^{1,2}$, Chong Chen ${ }^{3}$, Qilin $\mathrm{Li}^{1,2}$, Pengcheng Cai ${ }^{1}$, Zheng Wang ${ }^{2,4^{*}}$ (D) and Lin Wang ${ }^{1,2^{*}}$
}

\begin{abstract}
Background: A new coronavirus disease 2019 (COVID-19) has escalated to a pandemic since its first outbreak in Wuhan, China. A small proportion of patients may have difficulty in generating IgM or lgG antibodies against SARSCoV-2, and little attention has been paid to them.

Case presentations: We present two cases of confirmed COVID-19 patients and characterize their initial symptoms, chest $C T$ results, medication, and laboratory test results in detail (including RT-PCR, IgM/ IgG, cytokine and blood cell counts).

Conclusion: Both of patients with confirmed COVID-19 pneumonia failed to produce either IgM or IgG even 40 to 50 days after their symptoms onset. This work provides evidence demonstrating that at least a small proportion of patients may have difficulty in rapidly gaining immunity against SARS-CoV-2.
\end{abstract}

Keywords: Case report, COVID-19, IgM, IgG, Negative antibodies results

\section{Background}

During the outbreak of coronavirus 2019 (COVID-19) [1-3], a small proportion of confirmed COVID-19 patients fail to produce IgM or IgG antibodies against SARS-CoV-2 even 40 days or longer periods of time after onset of their initial symptoms. However, most of the current studies so far are focused on the general population but for these patients.

From January 30 to March 15, 310 of COVID-19 patients who were positive for SARS-CoV-2 real time reverse-transcription PCR (RT-PCR) testing and received IgM and IgG detection at Wuhan Union Hospital (Wuhan, China) were enrolled. RT-PCR was performed through amplifying ORF1ab gene and N gene of SARSCoV-2 (BioGerm, Shanghai, China) using oropharyngeal swab specimens of all patients. From March 4 to 15,

\footnotetext{
* Correspondence: zhengwang@hust.edu.cn; lin_wang@hust.edu.cn

${ }^{2}$ Research Center for Tissue Engineering and Regenerative Medicine, Union Hospital, Tongji Medical College, Huazhong University of Science and Technology, Wuhan, China

'Department of Clinical Laboratory, Union Hospital, Tongji Medical College, Huazhong University of Science and Technology, Wuhan, China

Full list of author information is available at the end of the article
}

IgM and IgG of SARS-CoV-2 were tested using blood samples for all these 310 patients. Two different kits were used to detect antibodies through immune colloidal gold (ICG) technique (Yingnuote, Tangshan, China) and chemiluminescence immunoassay (CLIA) technique (Yahuilong, Shenzhen, China). Laboratory test results were collected and analyzed.

Among 310 COVID-19-confirmed patients, 308 of them were tested positive for IgM and/ or IgG, but only two patients were negative for IgM and IgG detection.

\section{Case presentations \\ Case 1}

Patient 1 (Fig. 1), a 29-year-old man, developed a cough and a sore throat with no fever on January 28. Groundglass opacities in chest CT and positive RT-PCR test results were obtained on February 2 and 8, respectively. Four days later (February 12), this patient developed mild pneumonia and was hospitalized for treatment $(600 \mathrm{mg}$ of antiviral arbidol, orally, every $12 \mathrm{~h}$ ). From February 14 to 17 , four consecutive RT-PCR test results (every day) using his throat swab specimens were all 
negative. With remission of pneumonia symptoms and absorption of ground-glass opacities, the patient was discharged on February 21. However, on March 5, his RT-PCR test result was tested positive again during his follow-up, and he was hospitalized again in the next day. After two negative RT-PCR test results on March 10 and 12, the patient was discharged on March 15. No evidence showed that the patient's immune function was compromised (Table 1). Analyses of a large range of laboratory results revealed that most of tests were normal, including the Immunoglobulin G, M, A (IgG, IgM, IgA) and Complement 3, 4 (C3, C4) (Table S1). IgM and IgG were repeatedly tested using his serum samples by two different detection methods (see Methods for details) on March 7 and 8, which were all negative.

\section{Case 2}

Patient 2 (Fig. 1), a 58-year-old man, developed a fever and a cough on January 24 and were admitted to Wuhan Central Hospital on January 31. Multifocal ground-glass opacities were observed on chest CT images and the RT-PCR test for SARS-CoV-2 was positive on February 1. Despite anti-infection treatment and oxygen support, his symptoms worsened over the next few days, leading to severe pneumonia. On February 11 , the patient was transferred to Wuhan Union Hospital for further treatment $(400 \mathrm{mg}$ of moxiflxacin, daily; $1000 \mathrm{mg}$ of tienam, every $8 \mathrm{~h} ; 200 \mathrm{mg}$ of arbidol, every $8 \mathrm{~h} ; 40 \mathrm{mg}$ of methylprednisolone, every $12 \mathrm{~h}$ ).
The patient with glucocorticoid therapy showed reduced lymphocyte numbers and low ratios of $\mathrm{CD} 3$, CD4 and CD8 T cells (Table 1), suggesting his compromised immune functions. Elevated IL-6 level was related to his severe pneumonia as previously reported [4]. Moreover, 27 of 62 laboratory tests of this patient were abnormal, such as elevated levels of Creactive protein (CRP), neutrophils and lactate dehydrogenase (LDH), and decreased levels of albumin, and hemoglobin (Table S2). On February 17 and March 11, he received two consecutive RT-PCR tests, which were positive. However, IgM and IgG in his serum samples remained undetectable on March 4, 9, and 14 .

\section{Discussion and conclusions}

Serological tests have been widely utilized in the diagnosis of COVID-19. IgM could be detected as early as 1 day post- symptom onset (PSO) and was detectable in $85 \%$ of COVID-19 confirmed patients 7 days PSO [5]. As for IgG, over 90\% of COVID-19 confirmed patients produced this type of antibody 14 days after illness [5]. In this study, two patients with confirmed COVID-19 failed to produce either IgM or IgG even 40 to 50 days after their symptoms onset. Given that all of COVID-19 patients reportedly had sero-positive for IgM and IgG approximately 35 days after symptoms onset [6], the window period for antibody production in these two COVID-19 patients

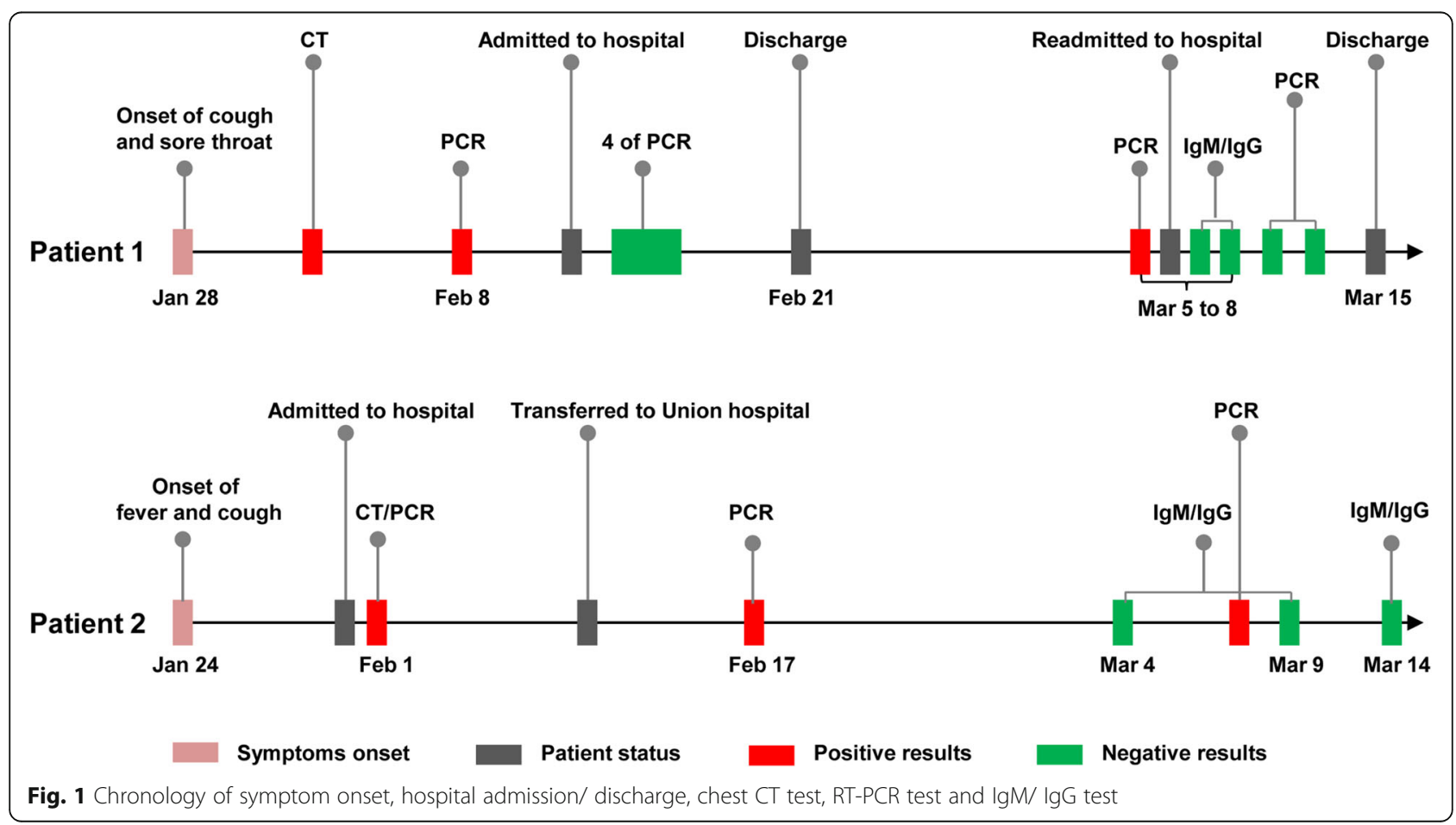


Table 1 Summary of Laboratory Examination Results of Two Patients

\begin{tabular}{llll}
\hline Laboratory test & Patient $\mathbf{1}$ & Patient 2 & Reference range \\
\hline Lymphocytes, 10 $/$ L & 1.87 & 0.62 & $1.1-3.2$ \\
CD3+ T cells, \% & 81.29 & 53.52 & $58.17-84.22$ \\
CD4+ T cells, \% & 40.14 & 34.79 & $25.34-51.37$ \\
CD8+ T cells, \% & 37.66 & 12.49 & $14.23-38.95$ \\
B cells, \% & 13.33 & 24.24 & $4.10-18.31$ \\
NK cells, \% & 4.10 & 18.98 & $3.33-30.47$ \\
CD4/CD8 & 1.07 & 2.79 & $0.41-2.72$ \\
IL-2, pg/mL & 3.93 & 4.04 & $0.10-4.10$ \\
IL-4, pg/mL & 3.56 & 3.56 & $0.10-3.20$ \\
IL-6, pg/mL & 4.41 & 681.69 & $0.10-2.90$ \\
IL-10, pg/mL & 3.95 & 10.08 & $0.10-5.00$ \\
TNF- $a, p g / m L$ & 37.11 & 3.29 & $0.10-23.00$ \\
IFN-, pg/mL & 3.58 & 3.14 & $0.10-18.00$ \\
IgG, au/mL & 2.13 & 3.05 & $0.00-10.00$ \\
IgM, au/mL & 3.21 & 2.67 & $0.00-10.00$ \\
\hline
\end{tabular}

may be much longer, possibly up to 50 days or even longer (if they eventually produce antibodies). These finding suggests that at least a small proportion of patients may have difficulty in rapidly gaining immunity against SARS-CoV-2. The cross-reactivity among different coronaviruses may lead to false-positive results in IgM and IgG detection [6], but few falsenegative outcomes were yielded, especially jointly using CLIA and ICG techniques. Thus, the fact that two or three times of negative results in the two patients using two different kits suggests that the results are unlikely to be false-negative. Analyses of the laboratory results showed that the 58-year-old patient (Case 2) had compromised immune functions, which might contribute to the negative IgM/ IgG results. However, the young patient (Case 1), who possessed normal immune functions and did not have any underlying disease, yet fails to produce IgM/ IgG 40 days after symptom onset. The reason remains unclear.

A further long-term follow-up should be carried out for these patients to determine whether they produce IgM/ IgG or persistently remain with no antibodies. Additionally, recurrence of COVID-19 was observed in the 29-year-old patient. If our finding of pneumonia recurrence in COVID-19 patients without antibodies is replicated, the management of these no-antibody patients needs to be more cautious as they may be prone to recurrence or re-infection. In summary, this work presents two typical cases of COVID-19 patients without producing IgM/ IgG and comprehensively characterizes their initial symptoms, chest $\mathrm{CT}$ results, medication, and laboratory test results, hoping to draw more attentions to this type of patients.

\section{Supplementary information}

Supplementary information accompanies this paper at https://doi.org/10. 1186/s12879-020-05419-3

Additional file 1: Table S1. Laboratory Examination Results of Patient 1. Table S2. Laboratory Examination Results of Patient 2.

\section{Abbreviations}

COVID-19: Coronavirus disease 2019; RT-PCR: Real-time polymerase chain reaction; CT: Computed tomography; IgG: Immunoglobulin G antibodies; IgM: Immunoglobulin M antibodies; ICG: Immune colloidal gold;

CLIA: Chemiluminescence immunoassay; PSO: Post- symptom onset

\section{Acknowledgements}

We thank Xueping Wang and colleagues from Immunoassay Team of Clinical Laboratory for generously providing antibodies results of COVID-19 patients. We thank Fenghua Chen, Zhi Geng, Yang Liu, Qianwen Zhang and colleagues from Nucleic Acid Testing Team of Clinical Laboratory for generously providing RT-PCR test results of COVID-19 patients.

Declarations

Not applicable.

\section{Authors' contributions}

JW, CC and PC contributed to data collection. JW, ZW, LW and QL

contributed to data analysis. All authors contributed to data interpretation. JW, ZW and LW drafted the manuscript. All authors critically commented on the manuscript and approved the final version.

\section{Funding}

No funding was received for this publication.

Availability of data and materials

The data-sets used during the current study are available from the corresponding author on reasonable request.

Ethics approval and consent to participate

This study was approved by the Wuhan Union Hospital of Huazhong University of Science and Technology institutional review board and informed consent were waived.

\section{Consent for publication}

Written informed consent was obtained from the individuals for the publication of any potentially identifiable images or data included in this article.

\section{Competing interests}

The authors declare that they have no conflicts of interest.

\section{Author details}

${ }^{1}$ Department of Clinical Laboratory, Union Hospital, Tongji Medical College, Huazhong University of Science and Technology, Wuhan, China. ${ }^{2}$ Research Center for Tissue Engineering and Regenerative Medicine, Union Hospital, Tongji Medical College, Huazhong University of Science and Technology, Wuhan, China. ${ }^{3}$ Department of Radiology, Union Hospital, Tongji Medical College, Huazhong University of Science and Technology, Wuhan, China. ${ }^{4}$ Department of Gastrointestinal Surgery, Union Hospital, Tongji Medical College, Huazhong University of Science and Technology, Wuhan, China.

Received: 5 June 2020 Accepted: 14 September 2020

Published online: 22 September 2020

\section{References}

1. Paules $\mathrm{Cl}$, Marston HD, Fauci AS. Coronavirus infections-more than just the common cold. JAMA. 2020;323(8):707-8. 
2. Li Q, Guan X, Wu P, et al. Early transmission dynamics in Wuhan, China, of novel coronavirus-infected pneumonia. N Engl J Med. 2020;382(13):1199-207.

3. Wang D, Hu B, Hu C, et al. Clinical characteristics of 138 hospitalized patients with 2019 novel coronavirus-infected pneumonia in Wuhan, China. JAMA. 2020;323(11):1061-9

4. Zhou F, Yu T, Du R, et al. Clinical course and risk factors for mortality of adult inpatients with COVID-19 in Wuhan, China: a retrospective cohort study. Lancet. 2020;395(10229):1054-62.

5. Theel ES, Slev P, Wheeler S, Couturier MR, Wong SJ, Kadkhoda K. The role of antibody testing for SARS-CoV-2: is there one? J Clin Microbiol. 2020;58(8): e00797-20.

6. Zhao J, Yuan Q, Wang H, et al. Antibody responsed to SARSCoV-2 in patients of novel coronavirus disease 2019. Clin Infect Dis. 2020

\section{Publisher's Note}

Springer Nature remains neutral with regard to jurisdictional claims in published maps and institutional affiliations.

Ready to submit your research? Choose BMC and benefit from:

- fast, convenient online submission

- thorough peer review by experienced researchers in your field

- rapid publication on acceptance

- support for research data, including large and complex data types

- gold Open Access which fosters wider collaboration and increased citations

- maximum visibility for your research: over $100 \mathrm{M}$ website views per year

At BMC, research is always in progress.

Learn more biomedcentral.com/submissions 